\title{
Absorção de 1-metilciclopropeno aplicado em maçãs 'Royal Gala' armazenadas em atmosfera refrigerada juntamente com madeira
}

\author{
Absorption of 1-metylcyclopropene applied on 'Royal Gala' apples stored in refrigerated air \\ together with wood
}

\author{
Auri Brackmann' ${ }^{\mathrm{I}}$ Vanderlei Both"I Josuel Alfredo Vilela Pinto ${ }^{\text {III }}$ Anderson Weber III \\ Elizandra Pivotto PavanelloII
}

\section{RESUMO}

Este trabalho teve por objetivo avaliar a interferência da madeira utilizada na construção das caixas, denominadas de bins, utilizadas no armazenamento comercial, sobre a eficiência da aplicação de doses de 1-metilciclopropeno (1-MCP) em maçãs da cultivar Royal Gala. Os tratamentos avaliados foram: controle (sem 1-MCP e sem madeira); 1 MCP (625nL L $\left.L^{-1}\right) ; 1-M C P\left(625 n L L^{-1}\right)+$ madeira de eucalipto (Eucaliptus sp); 1-MCP (625nL $\left.L^{-1}\right)+$ madeira de cedrinho (Erisma uncinatum Warm); 1-MCP $\left(1.250 n L L^{-1}\right) ; 1-M C P$ (1.250nL $\left.L^{-1}\right)+$ eucalipto e 1-MCP $\left(1.250 n L L^{-1}\right)+$ cedrinho. Após quatro meses de armazenamento a $0,5^{\circ} \mathrm{C}$ mais sete dias a $20^{\circ} \mathrm{C}$, não foi observada diminuição da qualidade dos frutos tratados com 1-MCP e armazenados na presença e ausência de madeira, evidenciando que as madeiras de eucalipto $e$ cedrinho não absorvem o 1-MCP a ponto de influenciar a eficiência do produto. A atividade da ACC oxidase, a síntese de etileno, a respiração, a firmeza da polpa e a cor da epiderme não diferiram entre as maçãs tratadas com 1-MCP $e$ armazenadas na presença ou não de madeira. Também não houve diferença entre as doses de 1.250nL $L^{-1}$ e 625nL $L^{-1}$ de 1MCP na maturação dos frutos. Conclui-se que os dois tipos de madeira utilizados na construção das embalagens e que são utilizados para o armazenamento de maçãs em câmaras frigoríficas comerciais não interferiram na eficiência de 1MCP sobre a maturação dos frutos.

Palavras-chave: ACC oxidase, etileno, qualidade.

\section{ABSTRACT}

This research aimed to evaluate the interference of the wood used in the boxes building, which the name are bins, used in the commercial storage, under the 1-metylcyclopropene (1-MCP) doses efficiency applied in 'Royal Gala' apples. The evaluated treatments were: control (without 1-MCP and without wood); 1-MCP (625nL L $\left.L^{-1}\right)$; 1-MCP (625nL $\left.L^{-1}\right)+$ eucalyptus wood (Eucaliptus sp); 1-MCP (625nL $\left.L^{-1}\right)+$ cedrinho wood (Erisma uncinatum Warm); 1-MCP (1.250nL $\left.L^{-1}\right)$; 1-MCP $\left(1.250 n L L^{-1}\right)+$ eucalyptus wood and 1-MCP $\left(1.250 n L L^{-1}\right)+$ cedrinho wood. After four months of cold storage at $0,5^{\circ} \mathrm{C}$ and more seven days at $20^{\circ} \mathrm{C}$, there was no decrease in the fruits quality that were treated with 1-MCP and stored on the presence and absence of wood, which showed that eucalyptus and cedrinho wood don't absorb 1-MCP to the point of influencing the product efficiency. The activity of the ACC oxidase, ethylene synthesis, respiration, flesh firmness and skin color didn't differ among the apples treated with 1-MCP and stored in the presence or absence of wood used in the bins building. There also was no difference among the doses of $1.250 \mathrm{~nL} \mathrm{~L} \mathrm{~L}^{-1}$ and $625 n L L^{-1} 1-M C P$ in the fruit maturation. In conclusion, the two kinds of wood used in the boxes building, which are used for the apple storage in commercial cold storage chambers, doesn't interfere in the efficiency of 1-MCP in apple maturation.

Key words: ACC oxidase, ethylene, quality.

\section{INTRODUÇÃO}

A rápida perda de qualidade da maçã 'Gala', bem como de suas mutantes, está relacionada à alta produção de etileno $\left(\mathrm{C}_{2} \mathrm{H}_{4}\right)$ e à respiração dos frutos (BRACKMANN, 1992). De maneira geral, quanto maior a produção e a ação do etileno, maior a velocidade com que ocorre o amadurecimento e a senescência dos frutos.

IDepartamento de Fitotecnia, Centro de Ciências Rurais (CCR), Universidade Federal de Santa Maria (UFSM), 97105-900, Santa

Maria, RS, Brasil. E-mail: auribrackmann@gmail.com.*Autor para correspondência.

${ }^{\text {II } C u r s o ~ d e ~ A g r o n o m i a, ~ C C R, ~ U F S M, ~ S a n t a ~ M a r i a, ~ R S, ~ B r a s i l . ~}$

IIIPrograma de Pós-graduação em Agronomia (PPGA), UFSM, Santa Maria, RS, Brasil. 
Atualmente, várias técnicas têm sido desenvolvidas para diminuir o efeito do etileno. A atmosfera controlada, com um baixo teor de oxigênio e alto nível de gás carbônico, reduz significativamente a produção e a ação do etileno (CHAMBROY \& SOUTY, 1994). Além disso, absorvedores de etileno podem ser usados para reduzir a concentração do gás dentro das câmaras frigoríficas com o objetivo de retardar a maturação de maçãs. A absorção do etileno, durante o armazenamento de maçãs 'Gala', permite a manutenção da firmeza de polpa, da acidez titulável e da cor de fundo da epiderme, além de diminuir a incidência de distúrbios fisiológicos (BRACKMANN et al., 2003). Há alguns anos, iniciou-se o uso comercial em maçãs do composto volátil 1-metilciclopropeno (1-MCP), que possui formulação em pó e, quando misturado a uma solução básica ou água, libera o ingrediente ativo em forma de gás, que atua por meio da ligação irreversível de suas moléculas aos receptores de etileno, localizados na membrana plasmática das células, impedindo assim respostas relativas a esse gás (BLANKENSHIP, 2001). O aumento da produção e da concentração interna de etileno e os eventos controlados por esse fitohormônio em frutos climatéricos são inibidos ou atrasados com a aplicação de 1-MCP(WATKINS, 2006).

A dose de $625 \mathrm{~nL} \mathrm{~L}^{-1}$ de 1-MCP aplicada no início do armazenamento apresenta grande eficácia na manutenção da qualidade da maçã 'Gala' durante seis meses de armazenamento refrigerado e mais 14 dias sob temperatura ambiente (BRACKMANN et al., 2004). No entanto, muitas vezes há divergências entre os resultados encontrados pelas pesquisas, que comprovam que o 1-MCP, além de restringir a ação do etileno, reduz sua produção e retarda o amadurecimento de frutos climatéricos, e os resultados práticos observados em empresas que armazenam comercialmente a maçã, onde, muitas vezes, os resultados demonstram uma menor eficiência do 1-MCP. Em diversas empresas de maçãs, a aplicação de 1-MCP mantém a firmeza de polpa apenas de quatro a oito Newtons acima dos frutos não tratados, enquanto que, em pesquisa laboratorial, essa diferença geralmente está acima de 10 Newtons, de acordo com BRACKMANN (2007 - informe verbal).

Poucas pesquisas têm sido realizadas com o objetivo de descobrir as causas dessa perda de eficiência de 1-MCP em câmaras frigoríficas comerciais. VALLEJO \& BEAUDRY (2006) demonstraram que a madeira e o papelão das embalagens removem rapidamente o 1-MCP na fase gasosa. Os autores sugerem que isso possa ser devido à absorção pela celulose, observando que as microfibrilas de celulose têm um vazio interno semelhante em tamanho à a- ciclodextrina utilizada para encapsulamento do 1-MCP na atual formulação SmartFresh ${ }^{\circledR}$. Tendo em vista que, no armazenamento de maçãs no Brasil, são utilizadas caixas de madeiras (bins), a diminuição da eficiência do 1-MCP, em câmaras comerciais, poderia estar relacionada com a absorção do produto por essa madeira.

Portanto, o presente trabalho objetivou avaliar a interferência da madeira utilizada na construção das caixas (bins) utilizadas no armazenamento comercial de maçãs sobre a eficiência do 1-MCP, aplicado em diferentes doses, em maçãs da cultivar 'Royal Gala'.

\section{MATERIAL E MÉTODOS}

O experimento foi conduzido no Núcleo de Pesquisa em Pós-colheita (NPP) do Departamento de Fitotecnia da Universidade Federal de Santa Maria com frutos da cultivar 'Royal Gala' provenientes de um pomar comercial do Município de Vacaria, colhidos no dia 10 de fevereiro de 2007. Antes do armazenamento, foram realizadas a seleção dos frutos, a homogeneização das amostras experimentais e a aplicação dos tratamentos. O delineamento experimental utilizado foi de blocos ao acaso, com quatro repetições e unidades experimentais compostas por 25 frutos. Todos os frutos foram acondicionados em caixas de plástico, dentro de minicâmaras experimentais de 232 litros, sob armazenamento refrigerado (AR), na temperatura de $0,5^{\circ} \mathrm{C}$, tolerando uma variação de $\pm 0,1^{\circ} \mathrm{C}$. A temperatura foi controlada automaticamente por termostatos, sendo monitorada diariamente, utilizando termômetros de mercúrio introduzidos na polpa de frutos. A umidade no interior das minicâmaras foi mantida em $96 \%$, com variação de $\pm 1 \%$, sendo monitorada com o auxílio de um psicrômetro.

Os frutos foram submetidos aos seguintes tratamentos: [1] AR (controle); [2] 1-MCP $\left(625 \mathrm{~nL} \mathrm{~L}^{-1}\right)$; [3] 1-MCP $\left(625 \mathrm{~nL} \mathrm{~L}^{-1}\right)+$ madeira de eucalipto (Eucaliptus sp); [4] 1-MCP (625nL L-1) + madeira de cedrinho (Erisma uncinatum Warm); [5] 1-MCP (1.250nL L $\left.{ }^{-1}\right)$; [6] 1-MCP $\left(1.250 \mathrm{~nL} \mathrm{~L}^{-1}\right)+$ madeira de eucalipto; [7] 1-MCP (1.250nL L'-1) + madeira de cedrinho.

Nos tratamentos com 1-MCP, utilizou-se o produto Smartfresh ${ }^{\circledR}(0,14 \%)$ nas concentrações de $625 \mathrm{~nL} \mathrm{~L}^{-1}$ e $1.250 \mathrm{~nL} \mathrm{~L}^{-1}$, com o objetivo de verificar se essas altas doses supririam a parte do produto que seria absorvida pela madeira. O produto foi solubilizado em $25 \mathrm{~mL}$ de água destilada e deionizada em recipiente hermético e, posteriormente, transferido para uma placa de Petri, já no interior da minicâmara, que foi imediatamente fechada. 
Nos tratamentos em que se utilizou madeira, esta foi previamente umedecida e cortada em tamanho de $0,25 \mathrm{~m} \times 1,25 \mathrm{~m}$ e acondicionada juntamente com as maçãs nas minicâmaras para receber o tratamento com 1-MCP. A relação da superfície da madeira, do volume da câmara e da massa de frutos do experimento foi equivalente àquela encontrada nas câmaras comerciais. Os frutos ficaram expostos ao tratamento com 1-MCP por 24 horas nas mesmas condições de temperatura e umidade descritas anteriormente e, em seguida, as minicâmaras foram ventiladas com o auxílio de uma bomba de sucção com vazão de $14 \mathrm{~m}^{3} \mathrm{~h}^{-1}$ durante uma hora. Os frutos de todo o experimento foram armazenados em quatro minicâmaras instaladas no interior de uma câmara frigorífica de $45 \mathrm{~m}^{3}$ e cada uma recebeu uma repetição de cada tratamento, onde permaneceram até as análises de maturação e qualidade.

As análises foram realizadas após quatro meses de armazenamento refrigerado e mais sete dias de exposição dos frutos a $20^{\circ} \mathrm{C}$, para simular o período de comercialização. Foram avaliadas as seguintes variáveis:

a) Produção de etileno: determinada por meio da produção desse gás por uma amostra de cerca de $1000 \mathrm{~g}$ de frutos, fechados hermeticamente em recipientes de cinco litros durante duas horas, a $20^{\circ} \mathrm{C}$. Após esse período, injetou-se $1 \mathrm{~mL}$ da atmosfera do espaço livre desses recipientes em um cromatógrafo a gás, marca Varian, modelo 3400, equipado com uma coluna de aço inox de 2,00m de comprimento, preparada com Porapak N80/100 e um detector de ionização de chama. Utilizou-se $\mathrm{N}$ como gás de arraste. As temperaturas da câmara ${ }^{2}$ de injeção, coluna e detector foram: $90^{\circ} \mathrm{C}, 140^{\circ} \mathrm{C} \mathrm{e} 200^{\circ} \mathrm{C}$, respectivamente. Os dados foram expressos em $\mu \mathrm{L} \mathrm{C}_{2} \mathrm{H}_{4} \mathrm{~kg}^{-1} \mathrm{~h}^{-1}$;

b) Respiração: determinada pela produção de $\mathrm{CO}_{2}$ por meio da leitura ar do espaço livre do mesmo recipiente que foi utilizado para determinação da produção de etileno, que foi circulado por meio de um analisador eletrônico de $\mathrm{CO}_{2}$, marca Agri-Datalog, sendo os dados expressos em $\mathrm{mL} \mathrm{CO}_{2} \mathrm{~kg}^{-1} \mathrm{~h}^{-1}$;

c) Cor de fundo da epiderme: determinada por meio de um colorímetro eletrônico da marca Minolta, modelo CR310, que utiliza o sistema de cores CIE L*a*b ou CIELAB, sendo as medições de coloração expressas em termos de valor L (variação da coloração de preta a branca), ângulo hue (mostra a localização da cor em um diagrama, em que o ângulo $0^{\circ}$ representa vermelho puro; $90^{\circ}$ representa o amarelo puro; $180^{\circ}$, o verde puro e $270^{\circ}$, o azul) e chroma (intensidade ou saturação de cor, sendo definida pela distância do ângulo hue no centro do diagrama tridimensional); d) Atividade da ACC oxidase: realizada aos sete dias a $20^{\circ} \mathrm{C}$, por meio da retirada de amostras de três gramas de casca da região equatorial dos frutos de cada unidade experimental. Estas foram imediatamente para uma solução contendo $0,1 \mathrm{mM}$ de ACC em $10 \mathrm{mM}$ do tampão MES (ácido 2 (N-morfolino) etanossulfônico), em pH 6,0. Após 30 minutos, as amostras foram acondicionadas em seringas herméticas de $50 \mathrm{~mL}$, nas quais adicionou-se $1 \mathrm{~mL}$ de $\mathrm{CO}_{2}$. Decorridos 30 minutos, a concentração de etileno presente nas seringas foi determinada, sendo os dados expressos em $\mu \mathrm{L} \mathrm{C}_{2} \mathrm{H}_{4} \mathrm{~kg}^{-1} \mathrm{~h}^{-1}$ (BUFLER, 1986).

e) Firmeza de polpa, sólidos solúveis totais (SST), acidez, podridões, degenerescência senescente, polpa farinácea e rachaduras foram avaliados conforme metodologia descrita por BRACKMANN \& SAQUET (1995).

Para cada variável avaliada, foi realizada uma análise da variância, sendo as médias comparadas pelo teste de Tukey, a 5\% de probabilidade de erro. As variáveis expressas em porcentagem foram transformada pela fórmula arco seno $\sqrt{x / 100}$, antes da análise da variância. Para testar a homocedasticidade de variâncias, utilizou-se o teste de Bartlett e as variáveis ACC oxidase, etileno e respiração não apresentavam variâncias homogêneas. Portanto, foram transformadas pela fórmula $\log _{10}(\mathrm{x}+1)$ e submetidas à nova análise de variância e a teste de médias.

\section{RESULTADOS E DISCUSSÃO}

Na análise da saída da câmara, não houve diferença significativa na síntese de etileno dos frutos dos tratamentos com 1-MCP, independentemente da dose, da presença e do tipo de madeira, apresentando valores inferiores à testemunha. Após sete dias a $20^{\circ} \mathrm{C}$, todos os tratamentos com aplicação de 1-MCP diferiram da testemunha, que apresentou um valor elevado de síntese de etileno (Tabela 1). As aplicações de maiores doses de 1-MCP (1.250 $\left.\mathrm{nL} \mathrm{L}^{-1}\right)$ reduziram as taxas de produção de etileno nos frutos, quando comparados com os frutos do tratamento que recebeu $625 \mathrm{~nL} \mathrm{~L}^{-1} \mathrm{e}$ que foi armazenado sem a presença de madeira. Porém, entre os tratamentos que receberam a mesma dose de 1-MCP não foi verificada diferença. Portanto, a madeira presente no interior das minicâmaras não influenciou a produção de etileno das maçãs, mesmo naquelas que receberam a menor dose de 1-MCP.

Da mesma forma que ocorreu com a produção de etileno, os frutos tratados com 1-MCP apresentaram atividade da enzima ACC oxidase estatisticamente inferior à testemunha (Tabela 1). Assim, 
Tabela 1 - Atividade da ACC oxidase, síntese de etileno e respiração das maçãs 'Royal Gala' em função da aplicação de doses de 1metilciclopropeno (1-MCP) associada ao armazenamento em presença ou não de madeira na câmara frigorífica, após quatro meses de armazenamento refrigerado seguido de sete dias a $20^{\circ} \mathrm{C}^{*}$. Santa Maria, RS, 2007.

\begin{tabular}{|c|c|c|c|c|c|}
\hline \multirow{2}{*}{ Tratamento } & \multirow{2}{*}{$\begin{array}{c}\text { ACC oxidase } \\
\left(\mu \mathrm{L} \mathrm{C}_{2} \mathrm{H}_{4} \mathrm{Kg}^{-1} \mathrm{~h}^{-1}\right)\end{array}$} & \multicolumn{2}{|c|}{ Etileno $\left(\mu \mathrm{L} \mathrm{C}_{2} \mathrm{H}_{4} \mathrm{Kg}^{-1} \mathrm{~h}^{-1}\right)$} & \multicolumn{2}{|c|}{ Respiração $\left(\mathrm{mL} \mathrm{CO}_{2} \mathrm{Kg}^{-1} \mathrm{~h}^{-1}\right)$} \\
\hline & & saída & sete dias & saída & sete dias \\
\hline Controle & $160,74 \mathrm{a}$ & $13,30 \mathrm{a}$ & $40,30 \mathrm{a}$ & $12,63 \mathrm{a}$ & $20,23 \mathrm{a}$ \\
\hline $\operatorname{MCP}\left(625 \mathrm{~nL} \mathrm{~L}^{-1}\right)$ & $18,35 \mathrm{~b}$ & $0,59 \mathrm{~b}$ & $5,96 \mathrm{~b}$ & $7,48 \mathrm{~b}$ & $16,18 \mathrm{abc}$ \\
\hline $\operatorname{MCP}\left(625 \mathrm{~nL} \mathrm{~L}^{-1}\right)$ e eucalipto & $15,74 \mathrm{~b}$ & $0,51 \mathrm{~b}$ & $4,80 \mathrm{bc}$ & $6,90 \mathrm{~b}$ & $17,17 \mathrm{ab}$ \\
\hline MCP $\left(625 n L^{-1}\right)$ e cedrinho & $16,97 \mathrm{~b}$ & $0,56 \mathrm{~b}$ & $4,52 \mathrm{bc}$ & $7,72 b$ & $15,81 \mathrm{abc}$ \\
\hline $\operatorname{MCP}\left(1250 \mathrm{~nL} \mathrm{~L}^{-1}\right)$ & $14,83 \mathrm{~b}$ & $0,23 \mathrm{~b}$ & $3,56 \mathrm{c}$ & $6,64 b$ & $12,86 \mathrm{abc}$ \\
\hline MCP $\left(1250 \mathrm{~nL} \mathrm{~L}^{-1}\right)$ e eucalipto & $20,82 \mathrm{~b}$ & $0,35 \mathrm{~b}$ & $3,59 \mathrm{c}$ & $8,11 \mathrm{~b}$ & $9,88 \mathrm{c}$ \\
\hline MCP $\left(1250 \mathrm{~nL} \mathrm{~L}^{-1}\right)$ e cedrinho & $18,46 \mathrm{~b}$ & $0,38 \mathrm{~b}$ & $3,59 \mathrm{c}$ & $7,93 \mathrm{~b}$ & $10,98 \mathrm{bc}$ \\
\hline CV $(\%)$ & 12,99 & 22,61 & 5,78 & 5,39 & 6,68 \\
\hline
\end{tabular}

*Tratamentos com médias não seguidas pela mesma letra na vertical diferem, pelo teste de Tukey, em nível de 5\% de probabilidade de erro.

podemos relacionar essa menor atividade da enzima com a menor produção de etileno nesses frutos, uma vez que o 1-MCP se liga aos receptores de etileno, diminuindo a atividade da enzima ACC oxidase e, consequentemente, a autocatálise de etileno. De acordo com BUFLER (1986), o etileno estimula um aumento da atividade da enzima ACC oxidase em tecidos da casca de maçã, ou seja, o etileno regula a sua autocatálise.

A respiração dos frutos apresentou um comportamento semelhante ao etileno na saída da câmara (Tabela 1 ). Aos sete dias a $20^{\circ} \mathrm{C}$, os frutos que receberam $1.250 \mathrm{~nL} \mathrm{~L}^{-1} \mathrm{e}$ foram armazenados com madeira de eucalipto apresentaram os menores níveis de respiração, entretanto diferiram significativamente apenas da testemunha e do tratamento que recebeu $625 \mathrm{~nL} \mathrm{~L}^{-1}$ de 1-MCP e foi armazenado com eucalipto. A maior dose apresentou maior eficiência no controle da respiração. Porém, não se pode afirmar que houve interferência da madeira na eficiência do 1-MCP, uma vez que, no tratamento com igual dose desse composto e sem madeira, a respiração dos frutos não apresentou diferença significativa.

A aplicação do 1-MCP em maçãs 'Royal Gala' manteve maior firmeza de polpa dos frutos, tanto naqueles tratamentos sem madeira, quanto naqueles com eucalipto ou cedrinho, quando comparados com o controle (Tabela 2). BRACKMANN et al. (2004), executando um experimento em que utilizaram três doses de 1-MCP $\left(0,312\right.$ e $\left.625 \mathrm{~nL} \mathrm{~L}^{-1}\right)$, com aplicação no início do armazenamento e reaplicação aos quatro meses, constataram que a melhor dose para reduzir o metabolismo da maçã 'Gala' é de $625 \mathrm{~nL} \mathrm{~L}^{-1}$, sem a necessidade de reaplicação. Dessa forma, neste experimento, as doses de $1-\mathrm{MCP}$ de $625 \mathrm{~nL} \mathrm{~L}^{-1}$ - dose recomendada - e $1.250 \mathrm{~nL} \mathrm{~L}^{-1}$ foram testadas com o objetivo de verificar se essa maior dose seria capaz de suprir o 1-MCP absorvido pela madeira contida nas minicâmaras e, assim, manter a qualidade dos frutos. No entanto, não foi verificada diferença de firmeza de polpa entre os frutos que receberam as diferentes doses, sugerindo que não houve interferência da madeira no efeito do 1-MCP. A maior firmeza de polpa das maçãs que receberam tratamento com 1-MCP pode estar diretamente relacionada à redução na produção de etileno desses frutos. Dependendo do tratamento, o 1-MCP manteve a firmeza de polpa de 14 a 17 Newtons maior do que nos frutos não tratados, enquanto que, em câmaras frigoríficas comerciais, o 1-MCP apenas mantém a firmeza de quatro a oito Newtons acima de frutos não tratados. Isso significa que existe um fator responsável pela redução da eficiência do produto em câmaras frigoríficas comerciais.

A acidez titulável mostrou comportamento semelhante entre os frutos que receberam 1-MCP, independentemente da dose, sendo apenas possível verificar diferença estatística entre os tratamentos com eucalipto em doses diferentes de 1-MCP, observandose menor acidez nos frutos que receberam a dose maior (Tabela 2). Os frutos que não receberam 1-MCP (controle) apresentaram acidez estatisticamente inferior aos demais, provavelmente porque essa condição proporcionou uma maior degradação dos ácidos orgânicos pelo processo respiratório, uma vez que esses frutos estavam submetidos a níveis mais altos de etileno, bem como apresentaram maior taxa respiratória (Tabela 1).

Após quatro meses de armazenamento na temperatura de $0,5^{\circ} \mathrm{C}$ e mais sete dias a $20^{\circ} \mathrm{C}$, a aplicação 
Tabela 2 - Acidez, firmeza de polpa e cor da epiderme das maçãs 'Royal Gala' em função da aplicação de doses de 1-metilciclopropeno (1$\mathrm{MCP}$ ) associada ao armazenamento em presença ou não de madeira na câmara frigorífica, após quatro meses de armazenamento refrigerado seguido de sete dias a $20^{\circ} \mathrm{C}^{*}$. Santa Maria, RS, 2007.

\begin{tabular}{|c|c|c|c|c|}
\hline \multirow{2}{*}{ Tratamento } & \multirow{2}{*}{$\begin{array}{c}\text { Acidez } \\
\left(\text { meq } 100 \mathrm{~mL}^{-1}\right)\end{array}$} & \multirow{2}{*}{$\begin{array}{l}\text { Firmeza } \\
\qquad(\mathrm{N})\end{array}$} & \multicolumn{2}{|c|}{ - } \\
\hline & & & $\mathrm{C}$ & $\mathrm{h}^{\mathrm{o}}$ \\
\hline Controle & $3,05 \mathrm{c}$ & $62,27 \mathrm{~b}$ & $48,25 \mathrm{a}$ & $81,82 \mathrm{a}$ \\
\hline $\operatorname{MCP}\left(625 \mathrm{~nL} \mathrm{~L}^{-1}\right)$ & $3,79 \mathrm{ab}$ & $76,12 \mathrm{a}$ & $45,67 \mathrm{a}$ & $81,63 \mathrm{a}$ \\
\hline MCP $\left(625 \mathrm{~nL} \mathrm{~L}^{-1}\right)$ e eucalipto & $3,88 \mathrm{a}$ & $79,20 \mathrm{a}$ & $45,51 \mathrm{a}$ & $84,02 \mathrm{a}$ \\
\hline $\operatorname{MCP}\left(625 \mathrm{~nL} \mathrm{~L}^{-1}\right)$ e cedrinho & $3,70 \mathrm{ab}$ & $78,57 \mathrm{a}$ & $45,69 \mathrm{a}$ & $81,85 \mathrm{a}$ \\
\hline $\operatorname{MCP}\left(1250 \mathrm{~nL} \mathrm{~L}^{-1}\right)$ & $3,78 \mathrm{ab}$ & $78,42 \mathrm{a}$ & $45,80 \mathrm{a}$ & $84,21 \mathrm{a}$ \\
\hline MCP $\left(1250 \mathrm{~nL} \mathrm{~L}^{-1}\right)$ e eucalipto & $3,58 \mathrm{~b}$ & $77,92 \mathrm{a}$ & $45,95 \mathrm{a}$ & $83,43 \mathrm{a}$ \\
\hline MCP $\left(1250 \mathrm{~nL} \mathrm{~L}^{-1}\right)$ e cedrinho & $3,66 \mathrm{ab}$ & $76,15 \mathrm{a}$ & $45,98 \mathrm{a}$ & $83,53 \mathrm{a}$ \\
\hline $\mathrm{CV}(\%)$ & 3,21 & 3,79 & 3,31 & 2,77 \\
\hline
\end{tabular}

*Tratamentos com médias não seguidas pela mesma letra na vertical diferem, pelo teste de Tukey, em nível de 5\% de probabilidade de erro.

de 1-MCP não interferiu na cor de fundo da epiderme dos frutos, uma vez que não houve diferença significativa quanto ao ângulo hue e chroma (Tabela 2). Da mesma forma, a aplicação de 1-MCP não influenciou a ocorrência de podridões (Tabela 3). Segundo EL-KAZZAZ et al. (1985), o etileno pode exibir um efeito estimulatório sobre o crescimento de alguns fungos e, consequentemente, aumentar a incidência e a severidade dos danos. Por outro lado, o etileno pode induzir a formação de fitoalexinas ou outros compostos que aumentam a resistência dos frutos aos patógenos. As variáveis degenerescência senescente, rachadura e polpa farinácea também não foram influenciadas pelos tratamentos (Tabela 3).

$\mathrm{Na}$ literatura, há poucos trabalhos sobre a interferência dos materiais utilizados em câmaras de armazenamento de frutas na absorção de 1-MCP. VALLEJO \& BEAUDRY (2006) verificaram que, quando foi aplicado 1-MCP em maçãs e estas foram armazenadas sozinhas, ocorria consumo de 50\% do 1MCP contido na câmara de armazenamento em aproximadamente 13 horas. No entanto, a adição de carvalho úmido resultou em um consumo de $90 \%$ da concentração inicial de 1-MCP em apenas seis horas. Assumindo a mesma taxa de perda, no caso de uma concentração inicial de $1 \mu \mathrm{L} \mathrm{L}^{-1}$, haveria apenas $0,1 \mu \mathrm{L}$ $\mathrm{L}^{-1}$ disponível após seis horas. Esses autores sugerem que bins de madeira úmida podem reduzir a concentração do 1-MCP para níveis poucos efetivos. Em trabalho semelhante, CALVO \& SOZZI (2009) concluíram que bins de madeira úmida podem comprometer a eficiência do 1-MCP no controle da ação do etileno sobre peras da cultivar Bartlett.

As respostas de maçãs ao 1-MCP variam de acordo com as cultivares (FAN et al., 1999; WATKINS et al., 2000). 'Gala', 'Ginger Gold' e 'Mc Intosh', por exemplo, diferem no grau e na duração das respostas ao 1-MCP. Possivelmente, este seja um dos motivos

Tabela 3 - Porcentagem de podridão, degenerescência senescente, rachadura e polpa farinácea em maçãs 'Royal Gala' em função da aplicação de doses de 1-metilciclopropeno (1-MCP) associada ao armazenamento em presença ou não de madeira na câmara frigorífica, após quatro meses de armazenamento refrigerado seguido de sete dias a $20^{\circ} \mathrm{C}^{*}$. Santa Maria, RS, 2007.

\begin{tabular}{lcccc}
\hline Tratamento & Podridão (\%) & $\begin{array}{c}\text { Degenerescência } \\
\text { senescente (\%) }\end{array}$ & Rachadura (\%) & Polpa farinácea (\%) \\
\hline Controle & $2,0 \mathrm{a}$ & $2,0 \mathrm{a}$ & $0,0 \mathrm{a}$ & $0,0 \mathrm{a}$ \\
MCP $\left(625 \mathrm{~nL} \mathrm{~L} \mathrm{~L}^{-1}\right)$ & $1,0 \mathrm{a}$ & $1,0 \mathrm{a}$ & $0,0 \mathrm{a}$ & $0,0 \mathrm{a}$ \\
MCP $\left(625 \mathrm{~nL} \mathrm{~L}^{-1}\right)$ e eucalipto & $0,0 \mathrm{a}$ & $3,0 \mathrm{a}$ & $0,0 \mathrm{a}$ & $0,0 \mathrm{a}$ \\
MCP $\left(625 \mathrm{~nL} \mathrm{~L}^{-1}\right)$ e cedrinho & $0,0 \mathrm{a}$ & $1,9 \mathrm{a}$ & $0,0 \mathrm{a}$ & $0,0 \mathrm{a}$ \\
MCP $\left(1250 \mathrm{~nL} \mathrm{~L}^{-1}\right)$ & $1,0 \mathrm{a}$ & $1,0 \mathrm{a}$ & $0,0 \mathrm{a}$ & $1,0 \mathrm{a}$ \\
MCP $\left(1250 \mathrm{~nL} \mathrm{~L}^{-1}\right)$ e eucalipto & $2,0 \mathrm{a}$ & $2,0 \mathrm{a}$ & $1,0 \mathrm{a}$ & $2,0 \mathrm{a}$ \\
MCP $\left(1250 \mathrm{~nL} \mathrm{~L}^{-1}\right)$ e cedrinho & $1,0 \mathrm{a}$ & $2,9 \mathrm{a}$ & $0,0 \mathrm{a}$ & 47,95 \\
$\mathrm{CV}(\%)$ & 74,41 & 63,19 & 35,61 & \\
\hline
\end{tabular}

*Tratamentos com médias não seguidas pela mesma letra na vertical diferem, pelo teste de Tukey, em nível de 5\% de probabilidade de erro.

Ciência Rural, v.39, n.6, set, 2009. 
pelos quais não foram obtidos, neste trabalho, resultados semelhantes aos citados anteriormente ou, também, por causa da diferença entre as espécies de madeira utilizadas nos diferentes experimentos.

A redução da eficiência de 1-MCP, em câmaras comerciais, também pode estar vinculada a fatores como estádio de maturação e tempo e método de resfriamento dos frutos. A umidade relativa na câmara de atmosfera controlada e o tempo transcorrido entre a colheita e a instalação da atmosfera também podem interferir na eficiência do produto. $\mathrm{O}$ potencial de ligação do 1-MCP com a madeira pode sofrer variação em função do nível de umedecimento da madeira, no momento da aplicação de 1-MCP(VALLEJO \&BEAUDRY, 2006).

\section{CONCLUSÃO}

Os dois tipos de madeira (eucalipto e cedrinho), utilizados na confecção das embalagens para o armazenamento de maçãs em câmaras frigoríficas comerciais, não interferem na eficiência de 1-MCP sobre a maturação dos frutos da cultivar 'Royal Gala' armazenados durante quatro meses em atmosfera refrigerada.

\section{INFORME VERBAL}

BRACKMANN, A. Informe verbal. Departamento de Fitotecnia, CCR, Universidade Federal de Santa Maria, 97105900, Santa Maria-RS. E-mail: auribrackmann@gmail.com.

\section{REFERÊNCIAS}

BLANKENSHIP, S. M. Ethylene effects and the benefits of $1-$ MCP. Perishables Handling Quarterly, North Carolina, n. 108, 4p, 2001.

BRACKMANN, A. Produção de etileno, $\mathrm{CO}$ e aroma de cultivares de maçã. Revista Brasileira de Fruticultura Jaboticabal, v.14, n.1, p.103-108,1992.

BRACKMANN, A. et al. Armazenamento de maçã "Gala" em atmosfera controlada com remoção de etileno. Ciência Rural, Santa Maria, v.33, n.4, p.647-650, 2003. Disponível em: <http:/ /www.scielo.br/scielo.php?script $=$ sci_arttext\&pid $=$ S0103 84782003000400009>. Acesso em: 19 mar. 2009. Doi: $10.1590 / \mathrm{S} 0103-84782003000400009$

BRACKMANN, et al. Qualidade da maçã cv. Gala tratada com 1-metilciclopropeno. Ciência Rural, Santa Maria, v.34, n.5, p.1415-1420, 2004. Disponível em: <http://www.scielo.br/ s cielo.php? s cript $=$ s ci arttext \& pid $=$ S 0103 84782004000500014>. Acesso em: 19 mar. 2009. Doi $10.1590 / \mathrm{S} 0103-84782004000500014$
BRACKMANN, A.; SAQUET, A.A. Armazenamento de maçã cv. Gala em atmosfera controlada. Revista Brasileira de Agrociência, Pelotas, v.1, n.2, p.55-60, 1995. Disponível em: <http://www.ufpel.tche.br/faem/agrociencia/v1n2/ artigo01.pdf $>$. Acesso em: 19 mar. 2009.

BUFLER, G. Ethylene-promoted conversion of 1aminocyclopropane-1-carboxylic acid to ethylene in peel of apple at various stages of fruit development. Plant Physiology, Rockville, v.80, p.539-543, 1986. Disponível em: $<$ http://www.plantphysiol.org/cgi/reprint/80/2/539> Acesso em: 24 mar. 2009.

CALVO, G.; SOZZI, G.O. Effectiveness of 1-MCP treatment on 'Bartlett' pears as influenced by the cooling method and the bin material. Postharvest Biology and Technology, Amsterdam, v.51, n.1, p.49-55, 2009. Disponível em: <http:/ / $\mathrm{w}$ w w. $\mathrm{s}$ c i e $\mathrm{n}$ c e d i $\mathrm{r}$ e $\mathrm{c}$ t. science?_ob=ArticleURL\&_udi=B6TBJ-4T41PDV-1> Acesso em: 24 mar. 2009. Doi: 10.1016/j.postharvbio.2008.06.011.

CHAMBROY, Y.; SOUTY, M. Rôle du CO sur le comportement des fruits à noyau utilisation des atmosphéres modifiées. In. SEMINARIO CELEBRADO EN LA FIRA DE LLEIDA, 1994, Lleida - España. Actas... IRTA, CIHEAL y Ajuntament de Lleida, 1994. p.139-169.

EL-KAZZAZ, et al. Ethylene effects on postharvest fruit diseases. In: NATIONAL CONTROLLED ATMOSPHERE RESEARCH CONFERENCE, 4., 1985, Raleigh, North Carolina - USA. Procedings... Raleigh: North Carolina State University, 1985. V.1. p.348-351.

FAN, X. et al. Development of apple superficial scald, soft scald, core flush and greasiness is reduced by MCP. Journal of Agricultural and Food Chemistry, Washington, v.47, n.8, p.3063-3068, 1999. Disponível em: <http://pubs.acs.org/doi/ full/10.1021/jf981176b $>$ Acesso em: 24 mar. 2009. Doi: $10.1021 / \mathrm{jf} 981176 \mathrm{~b}$

VALLEJO, F.; BEAUDRY, R. Depletion of 1-MCP by 'nontarget' materials from fruit storage facilities. Postharvest Biology and Technology, Amsterdam, v.40, p.177-182, 2006. Disponível em: <http://www.sciencedirect.com/ science? ob=ArticleURL\& udi=B6TBJ-4JRKVHX-4> Acesso em: 24 mar. 2009. Doi: $10.1016 /$ j.postharvbio.2006.01.003.

WATKINS, et al. Responses of early, mid and late season apple cultivars to postharvest application of 1-methylcyclopropene (1-MCP) under air and controlled atmosphere storage conditions. Postharvest Biology and Technology, Amsterdam, v.19, p.1732, 2000. Disponível em: < http://www.sciencedirect.com/ science? ob=ArticleURL\& udi=B6TBJ-405TFWP-3> Acesso em: 24 mar. 2009. Doi: 10.1016/S0925-5214(00)00070-3.

WATKINS, C.B. The use of 1-methylcyclopropene (1-MCP) on fruits and vegetables. Biotechnology Advances, Oxford, v.24, p.389-409, 2006. Disponível em: < http:// www.sciencedirect.com/science?_ob=ArticleURL\&_udi=B6T4X4JFGFB4-1 > Acesso em: 24 mar. 2009. Doi: 10.1016 j.biotechadv.2006.01.005 . 